\title{
A METROLOGIA NA ÁREA DE SAÚDE: GARANTIA DA SEGURANÇA E DA QUALIDADE DOS EQUIPAMENTOS ELETROMÉDICOS
}

\author{
Elisabeth Costa Monteiro ${ }^{1}$ \\ Marcelo Lúcio Lessa ${ }^{1,2 .}$
}

\begin{abstract}
Resumo: Os grandes avanços da metrologia na área científica e industrial não têm paralelo na área da saúde, onde apesar da óbvia importância, sua aplicação é ainda incipiente. A acelerada evolução da tecnologia dos equipamentos médicos os tornou mais complexos, os caracterizando, atualmente, por um crescente número de medições de parâmetros fisiológicos. Tais equipamentos são essenciais para o diagnóstico, caracterização de riscos, tratamento e acompanhamento da evolução clínica de pacientes. O presente trabalho apresenta alguns dados da literatura sobre os riscos do uso de equipamentos para saúde sem garantia de confiabilidade metrológica; apresenta aspectos e limitações da legislação e normas referentes a equipamentos eletromédicos (EEM) no Brasil, discutindo-se o controle pré e pós-comercialização; e aponta outros fatores importantes para a garantia da confiabilidade metrológica como as acreditações voluntárias de Estabelecimentos Assistenciais de Saúde (EAS), a adequação da formação profissional para o gerenciamento de EEM, a disseminação da cultura metrológica e o desenvolvimento de uma rede de laboratórios capacitada para calibração e ensaio de EEM.
\end{abstract}

Palavras Chaves: Metrologia, Saúde, Equipamentos Eletromédicos.

Abstract: There is no parallel between the great metrology advancements observed in the scientific and industrial areas when compared to the health area. Although being obviously important, the application of metrology in health is still incipient. With its accelerated evolution, the medical equipment technology became more complex, measuring an increasing number of physiological parameters. These equipments are essential for diagnosis, risk analysis, treatment and clinical follow up. This work presents some data of literature concerning risks due to the use of medical devices without metrological reliability; presents aspects and limitations of laws, regulations and standards concerning medical electrical equipment (MEE) in Brazil, being discussed its control during pre-sale and lifetime use periods; and indicates other important factors for the guarantee of metrological reliability as voluntary accreditations of health care facilities, the adequate professional education and training for MEE management, the diffusion of metrological culture and the development of a qualified laboratory network for calibration and tests of MEE.

Key-words: Metrology, Health, Electromedical Equipments

\footnotetext{
${ }^{1}$ Pontifícia Universidade Católica do Rio de Janeiro - Programa de Pós-Graduação em Metrologia (PósMQI) beth@metrologia.ctc.puc-rio.br

${ }^{2}$ EngeClinic Serviços Ltda mllessa@engecliniconline.com.br

ENGEVISTA, v. 7, n. 2, p. 51-60, dezembro 2005 


\section{INTRODUÇÃO}

A metrologia avançou muito na área científica e industrial, mas, apesar da óbvia importância, sua aplicação é ainda incipiente na área da saúde.

$\mathrm{Na}$ área biomédica a realização de medições de parâmetros fisiológicos é essencial para o diagnóstico, caracterização de riscos, tratamento e acompanhamento da evolução clínica de pacientes. Neste sentido, os procedimentos de medição aplicados à área da saúde devem se caracterizar pela elevada exatidão e rastreabilidade ao Sistema Internacional de Unidades. Outros fatores importantes referente às medições na área da saúde consistem nas características de baixo custo, não-invasividade e inocuidade. Estes fatores vêm sendo perseguidos pelos trabalhos de pesquisa e desenvolvimento de inovações em instrumentação na área da saúde $[1,2]$.

O impressionante desenvolvimento da Ciência e Tecnologia (eletrônica e informártica) no século XX contribuiu para uma acelerada evolução dos equipamentos médicos, tornando-os mais complexos e dificultando a detecção de mal funcionamento ou erro de medição por parte dos operadores através da simples inspeção. Observa-se um crescente aumento do número de variáveis fisiológicas cuja medição vem se tornando disponível. Os médicos, por sua vez, passaram a confiar cada vez mais seus diagnósticos e tratamentos nos parâmetros quantitativos oferecidos por tais instrumentos. O risco do uso de equipamentos sem a adequada avaliação metrológica ganhou destaque somente após 1990 com a publicação dos resultados do Harvard Medical Practice Study sobre eventos adversos [3]. Como descrito mais adiante, o estudo de Harvard concluiu que $3,7 \%$ dos pacientes hospitalizados sofrem eventos adversos, sendo que $13,6 \%$ dos incidentes são mortais [3].

A confiabilidade metrológica dos equipamentos biomédicos com realização de calibrações rastreadas a padrões internacionais garante não somente a segurança dos diagnósticos e tratamentos, mas também a desejável comparabilidade internacional nas biomedições.

Os equipamentos biomédicos no Brasil, conforme sua classificação quanto ao potencial de risco à saúde de seus usuários (pacientes e/ou operadores), necessitam de registro da Agência Nacional de Vigilância Sanitária (ANVISA) para que os fabricantes os lancem no mercado consumidor. A exigência do registro da ANVISA, conforme será descrito, implica numa prévia certificação de conformidade a normas técnicas específicas, emitida por Organismos de Certificação de Produtos (OCP) acreditados pelo Instituto Nacional de Metrologia, Normalização e Qualidade Industrial (INMETRO). A Certificação da Conformidade resulta não somente na proteção da integridade física dos usuários, como também na implementação de um ciclo virtuoso entre os sistemas regulador e produtivo. A adaptação dos equipamentos e de seus fabricantes aos regulamentos técnicos estimula melhorias qualitativas nos produtos e processos de fabricação [4].

Após a cormercialização dos produtos médicos, com a exceção apenas do termômetro clínico de mercúrio em vidro e do esfigmomanômetro mecânico de medição não-invasiva do tipo aneróide, que possuem regulamentação do INMETRO, além dos EEM envolvendo a utilização de radiações ionizantes, não existe nenhuma lei ou regulamentação que torne compulsório o controle dos outros instrumentos biomédicos para a garantia da confiabilidade metrológica, com calibrações rastreadas durante o tempo de vida em uso.

Os custos adicionais de calibrações rastreadas são muito evidentes para a gestão e administração na área da saúde, ao passo que os benefícios como melhores resultados diagnósticos e terapêuticos para os pacientes são difíceis de quantificar economicamente.

O setor da saúde onde a metrologia foi aplicada mais precocemente é a área envolvida com a utilização de radiações ionizantes. Já em 1976, o Instituto de Radioproteção e Dosimetria (IRD) passou a 
fazer parte de uma rede coordenada pela Organização Mundial de Saúde (OMS) e a Agência Internacional de Energia Atômica (AIEA) para estabelecer a rastreabilidade da calibração de instrumentos aos laboratórios e clientes em hospitais e clínicas de tratamento do câncer. Embora ainda aquém da situação ideal, a área das radiações ionizantes permanece com o nível de controle da confiabilidade metrológica mais avançado do que as outras áreas da saúde.

Uma área biomédica que se encontra sob ascendente preocupação metrológica é a biotecnologia. Uma das recomendações da 21st Conférence Générale des Poids et de Mesures (CGPM), em 1999, foi a de que se estabelecesse uma infra-estrutura de medição internacional adequada para assegurar a rastreabilidade das medições na área de Biotecnologia. A metrologia nesta área é fundamental para a obtenção de resultados confiáveis e internacionalmente equivalentes de testes forenses, identificação genética, análise de produtos biotecnológicos e marcadores para diagnóstico clínico.

O presente trabalho apresenta uma revisão e diagnóstico da situação atual da garantia da confiabilidade metrológica para EEM no Brasil. São apresentados aspectos legislativos, normativos e discutidos alguns obstáculos que dificultam o acesso à garantia da confiabilidade metrológica de EEM

Objetivando levantar alguns subsídios sobre a importância da metrologia no ambiente da saúde, a seguir são apresentados exemplos descritos na literatura sobre o impacto dos erros de calibração nas decisões médicas e uma breve descrição sobre eventos adversos, uma das importantes consequências da não confiabilidade metrológica de EEM.

\section{IMPACTO DAS NÃO CONFORMIDADES METROLÓGICAS SOBRE AS DECISÕES MÉDICAS E A OCORRÊNCIA DE EVENTOS ADVERSOS}

ENGEVISTA, v. 7, n. 2, p. 51-60, dezembro 2005
A medicina moderna estabelece consensos e estratégias para tratamento das enfermidades mais comuns assumindo, no entanto, que todos os laboratórios de análise e os equipamentos utilizados na obtenção de diagnósticos através da medição de parâmetros operam de forma uniforme, apresentando os resultados das medições com a confiabilidade metrológica necessária, o que, conforme será descrito, não é garantido.

Segue uma revisão de alguns resultados descritos na literatura, que ilustram, através do exemplo da medição da pressão arterial, parâmetro fisiológico mais medido no mundo, a importância da confiabilidade metrológica na área de saúde.

A pressão arterial é um fator de risco para doenças coronárias e cardiovasculares. A hipertensão arterial é uma das poucas doenças diagnosticadas pela simples medição de um único parâmetro fisiológico.

A medição da pressão arterial pode ser efetuada por vários tipos de esfigmomanômetros, sendo que para um destes, o esfigmomanômetro mecânico de medição não-invasiva do tipo aneróide, já existe regulamentação técnica metrológica (RTM) elaborada pelo INMETRO. Neste procedimento é permitido um erro estático não superior a $\pm 3 \mathrm{mmHg}[5,6]$. No entanto, um estudo realizado na Austrália, constatou que, a utilização de um esfigmomanômetro que superestime a pressão diastólica em 3 mmHg resulta em um aumento de $83 \%$ no número de pacientes com pressão arterial diastólica superior a $95 \mathrm{mmHg}$, ou seja, para cada 5 pacientes corretamente diagnosticados como hipertensos, outros 4 seriam erroneamente diagnosticados como hipertensos. Por outro lado, caso o esfigmomanômetro subestime a pressão disatólica em $3 \mathrm{mmHg}$, quase metade dos pacientes com pressão diastólica superior a 95 mmHg não seriam identificados [7]. Os resultados obtidos pelos pesquisadores Rouse e Marshal (2001) corroboram tal preocupação e sugerem que a tolerância de $\pm 3 \mathrm{mmHg}$ é muito ampla para a realização de diagnósticos confiáveis de hipertensão arterial diastólica, indicando $1 \mathrm{mmHg}$ 
como o valor ideal para a incerteza da medição [10].

Um estudo realizado no Reino Unido e publicado em 2002 mostrou que 28\% dos esfigmomanômetros de mercúrio e $42 \%$ dos aneróides apresentam erro superior a 4 mmHg. O estudo revela que apenas um em cada 54 médicos possui o cuidado de manutenir e calibrar seus esfigmomanômetros [8].

No Brasil, em 1997 o INMETRO realizou um estudo de verificação da calibração dos esfigmomanômetros em uso em hospitais localizados em Juiz de Fora, São Paulo e Rio de Janeiro. Os resultados do trabalho indicaram que $61 \%$ dos esfigmomanômetros verificados apresentaram erros acima de $4 \mathrm{~mm} \mathrm{Hg} \mathrm{[9].}$

A partir dos dados acima descritos, referentes a um instrumento de medição simples, pode-se prever que a sofisticada tecnologia disponível na atualidade para a área da saúde, apesar dos evidentes benefícios, possui grandes riscos de iatrogenias e ocorrência de Eventos Adversos (EA). Estes últimos são definidos como as complicações que acometem os pacientes hospitalizados e que não são decorrentes da evolução natural da enfermidade que os levou a serem hospitalizados.

A Organização Mundial de Saúde (OMS), na $55^{\mathrm{a}}$ Assembléia Geral, divulgou uma série de estudos realizados nos EUA, na Austrália, no Reino Unido (Inglaterra, Escócia, Pais de Gales e Irlanda do Norte) e na Dinamarca [11]. A finalidade dos estudos apresentados era mapear a ocorrência de EA no universo de pacientes hospitalizados. Dentre os estudos apresentados, destacou-se o realizado na Universidade de Harvard. Este último estabeleceu padrões para a quantificação dos EA. Os resultados do estudo de Harvard indicaram que num total de 30.195 internações, foram verificados $1.133 \mathrm{EA}$, o que corresponde a 3,8 \% de todos os pacientes internados. Detalhando ainda mais o estudo, concluiu-se que, do total de vítimas de EA, 74 \% foram acometidos de alguma incapacidade temporária e $14 \%$ evoluíram a óbito [3, 11]. Esses números impressionam se considerarmos que no Brasil, entre outubro de 2003 e setembro de 2004, o Sistema Único de Saúde (SUS), internou 11.561.118 [12]. Projetando a realidade de Harvard para os dados do SUS, poderíamos esperar a ocorrência de $462.445 \mathrm{EA}$, dos quais 323.711 gerariam incapacidade temporária e 64.742 óbitos. Este número é superior ao número de óbitos relacionados a acidentes envolvendo veículos automotivos ou mesmo decorrentes da AIDS [12].

Apesar de vários fatores serem responsáveis pela ocorrência de EA os EEM não-conformes são considerados uma importante fonte de risco. Ainda na $55^{\mathrm{a}}$ Assembléia Geral, a OMS fez um alerta especial aos países em desenvolvimento e subdesenvolvidos, onde a escassez de recursos, a falta de qualificação técnica do pessoal envolvido, a falta de uma infraestrutura adequada nos estabelecimentos assistenciais de saúde (EAS) e a incipiente legislação própria para o setor, pode agravar a situação [11]. Neste alerta, a OMS estima ainda que cerca de $50 \%$ dos EEM disponíveis nestes países estejam operando fora dos padrões recomendados pelos fabricantes [11].

A seguir é apresentada uma breve descrição da situação da regulação de EEM no Brasil. A regulação de tais produtos é uma ferramenta essencial para a garantia da confiabilidade metrológica.

\section{REGULAÇÃO DOS EEM}

O controle de EEM foi estabelecido por legislação relativamente recente. A Lei 6.360, de 23 de setembro de 1976, submeteu ao sistema de vigilância sanitária os EEM e outros produtos médicos, incluindo os produtos importados, tornando estes impedidos de industrialização, exposição à venda ou entrega ao consumo antes de registrados no Ministério da Saúde. Esta Lei 6.360 foi regulamentada pelo Decreto 79.094, de 5 de janeiro de 1977, que estabeleceu os requisitos para o registro dos produtos médicos. A Portaria n 2.043 do Ministério da Saúde, de 12 de dezembro de 1994, definiu o universo de produtos sujeitos ao sistema da qualidade, 
classificando-os quanto ao potencial de risco à saúde de pacientes e/ou operadores, tendo adotado o Sistema Nacional de Metrologia, Normalização e Qualidade Industrial (Sinmetro) para verificação e comprovação da conformidade desses produtos. Através da Lei 9.782, de 26 de janeiro de 1999, foi criada a Agência Nacional de Vigilância Sanitária (ANVISA), que vinculada ao Ministério da Saúde, incorporou as competências da Secretaria de Vigilância Sanitária do Ministério da Saúde além de outras missões. A Resolução $n^{0} 444$ de 31 de agosto de 1999 da ANVISA estabeleceu o modelo adotado para garantir a segurança sanitária dos equipamentos eletromédicos (Autorização de Modelo), adotando as Normas Técnicas da Série NBR IEC 60.601-1: Equipamento Eletromédico. Parte I - Prescrições Gerais Para Segurança; e Normas Técnicas particulares da Série NBR IEC 60.601-2. Com a RDC 59, de 27 de junho de 2000, ficou estabelecido para todos os fornecedores de produtos médicos, o cumprimento dos requisitos estabelecidos pelas "Boas Práticas de Fabricação de Produtos Médicos”.

Com relação ao uso de tecnologias envolvendo as radiações ionizantes, desde 1988, a Comissão Nacional de Energia Nuclear (CNEN) definiu requisitos técnicos compulórios para radioproteção através da norma CNEN - NE-3.01, tendo estabelecido Requisitos de Radioproteção e Segurança especificamente para Serviços de Medicina Nuclear e Radioterapia respectivamente em 1989 e 1990 através das normas CNEN-NN-3.05 e CNEN-NE3.06. No entanto, uma legislação referente à proteção radiológica em radiodiagnóstico médico e odontológico só foi estabelecida em Junho de 1998 pelo Ministério da Saúde e a Secretaria de Vigilância Sanitária através da Portaria $\mathrm{N}^{0} 453$, onde também constam requisitos técnicos para a implementação da garantia da confiabilidade metrológica destes equipamentos $[13,14]$.

\subsection{Normas Técnicas para EEM}

ENGEVISTA, v. 7, n. 2, p. 51-60, dezembro 2005
A Associação Brasileira de Normas Técnicas (ABNT) vem elaborando normas brasileiras e adaptando normas internacionais. Especificamente para EEM, atualmente a ABNT possui 59 normas, sendo 12 puramente elaboradas no Brasil (NBR), 40 adaptadas de normas elaboradas pela International Electrotechnical Commission (NBRIEC) e 7 adaptadas de normas elaboradas pela International Organization for Standardization (NBRISO). Por serem apenas normas técnicas, não são compulsórias isoladamente, a Resolução $\mathrm{n}^{\circ} 444$ de 1999 da ANVISA as tornam compulsórias para certificação de conformidade e obtenção de registro antes do lançamento do EEM no mercado. Com referência específica a EEM envolvendo radioações ionizantes, são também utilizadas 3 regulamentações técnicas elaboradas pelo CNEN, a Portaria 453 de 1998 do Ministério da Saúde e a Resolução RE $\mathrm{n}^{0} 64$ contendo detalhes técnicos e publicada pela ANVISA em 2003.

A primeira norma elaborada pela ABNT envolvendo segurança para EEM (NBR9153) foi publicada em 1985 e a primeira norma ABNT adaptada da série IEC 60601 foi publicada em 1994 (NBR IEC 60601), tendo o correspondente documento da IEC sido publicado em 1977.

No entanto, à exceção das regulamentações técnicas para EEM envolvendo radiações ionizantes, em geral, estas normas possuem prescrições apenas para aspectos de segurança de EEM. Somente recentemente a International Electrotechnical Commission (IEC) começou a elaborar normas com precrições para avaliação de desempenho de EEM. As normas IEC para desempenho compõem a Série IEC 606013-xx. A IEC publicou uma primeira norma com prescrições para desempenho, que já foi adaptada pela ABNT, e uma outra norma técnica para desempenho se encontra em projeto:

- NBR IEC 60601-3-1: Equipamento eletromédico - Parte 3-1: Prescrições de desempenho essencial para equipamento de monitorização da pressão parcial 
transcutânea de oxigênio e de dióxido de carbono

- IEC 60601-3-2: Performance of Recording and Analyzing Single Channel and Multichannel Electrocardiographs. (Projeto)

Atualmente verifica-se o nascimento da preocupação internacional com o estabelecimento de normas que visam o controle de produtos médicos no período após a comercialização. A seguir são listadas três normas em fase final de elaboração pela International Electrotechnical Commission (IEC), que têm por objetivo o estabelecimento de procedimentos para a realização de testes recorrentes e testes após reparo:

- IEC 62354 - General Testing Procedures for Medical electrical equipment: (Technical Report).

- IEC 62353 - Medical Eletrical Equipment - Recurrent Test and Test After Repair of Medical Electrical Equipment.

- IEC 62366 - Medical Devices General Requirements for Safety and Performance - Usability.

\subsection{Controle de EEM em uso}

No Brasil, assim como em muitos outros países do mundo, uma atenção especial é dedicada à certificação de produtos para a saúde antes de comercializá-los, denotando uma preocupação especial com a qualidade de tais produtos no momento prévio ao lançamento no mercado consumidor.

Neste sentido, com a exceção dos equipamentos de radioterapia e radiodiagnóstico, que possuem legislação através das normas CNEN e da portaria 453 que obrigam 0 controle metrológico durante a vida útil, o restante dos EEM não possui obrigatoriedade quanto à avaliação da confiabilidade metrológica após sua comercialização.

A Organização Internacional de Metrologia Legal (OIML) elabora recomendações metrológicas sobre as quais, em geral, se baseiam as regulamentações técnicas metrológicas (RTM) elaboradas pelo INMETRO. A RTM tem por finalidade colocar sob o controle do Estado diferentes categorias de instrumentos de medição, fixando requisitos técnicos e metrológicos para utilização e verificação [15]. As exigências das RTM conduzem ao controle metrológico dos instrumentos de uma forma mais ampla, tanto antes da entrada do produto médico no mercado, através da aprovação de modelo e verificação inicial, quanto durante o período da vida útil do mesmo, através da verificação periódica e eventual.

A seguir está listada uma relação das Recomendações Internacionais da OIML para a área da saúde [15]:

- R7 - Termômetros clínicos de vidro, de mercúrio, com dispositivo de máxima

- R16 - Esfigmomanômetros - Parte 1 e Parte 2

- R26 - Seringas médicas

- R78 - Pipetaswestergrenpara medição da velocidade de sedimentação das hemácias

- R89 -Eletroencefalógrafos

- R90 - Eletrocardiógrafos

- R93 - Focômetros

- R114 - Termômetros clínicos, elétricos, com medição contínua (CTI)

- R115 - Termômetros clínicos, elétricos, com dispositivo de máxima

- R122 - Aparelhos para audiometria vocal

- R128 - Bicicleta ergométrica

A Inglaterra e a Alemanha são os países que incorporaram o maior número de recomendações OIML para a área da saúde. Atualmente, o Brasil possui duas RTM na Área de Saúde (termômetro clínico de mercúrio em vidro e o esfigmomanômetro mecânico de medição não-invasiva do tipo aneróide), e grupos de trabalho no INMETRO estão atuando para adaptar outras recomendações para a área da saúde 
referentes aos instrumentos de medição que se encontram listados a seguir [16]:

- Esfigmomanômetros digitais

- Esfigmomanômetros de coluna de mercúrio

- Termômetros clínicos digitais

- Eletrocardiógrafos

- Eletroencefalógrafos

Objetivando a garantia de segurança sanitária de produtos para saúde, além da mencionada atuação da ANVISA de controle do produto médico no momento de seu lançamento no mercado consumidor, a agência atua no período de póscomercialização através da Tecnovigilância.

A atuação da agência no momento prévio à comercialização do produto se realiza através da exigência do registro ANVISA, que implica na apresentação de certificado de conformidade emitido por organismo acreditado no Sistema Brasileiro de Certificação (SBC). Por outro lado, no período de pós-comercialização a ANVISA atua através da Tecnovigilância, onde se realiza monitoramento das notificações de eventos adversos enviadas pelos EAS.

\section{ACREDITAÇÃO VOLUNTÁRIA DE EAS}

$\mathrm{Na}$ presente conjuntura, a acreditação voluntária de EAS consiste no grande promotor da confiabilidade metrológica de EEM em uso.

As acreditações são realizadas através de entidades nacionais ou internacionais, destacando-se a International Standardization Organization (ISO), a Organização Nacional de Acreditação (ONA), a Joint Comission for Acreditation on Health Organizations (JCAHO), a American Association of Blood Banks (AABB), etc. Tais organismos estabelecem exigências aos estabelecimentos acreditados no que se refere à garantia da confiabilidade dos EEM, sobretudo no que diz respeito a calibrações rastreadas e manutenções preventivas e corretivas. Atualmente existem no Brasil cerca de 40 hospitais acreditados e vários outros estão em processo de acreditação.

A garantia da confiabilidade metrológica na área da saúde por meio de acreditações voluntárias se estende não só à rede hospitalar, em particular, mas também às organizações prestadoras de serviços de Hemoterapia, de Nefrologia e Terapia Renal Substitutiva e de Laboratórios Clínicos. Estes últimos também têm vários programas de acreditação disponíveis e vem se observando um significativo crescimento do número de laboratórios clínicos acreditados.

\section{LABORATÓRIOS PARA CALIBRAÇÃO E ENSAIO DE EEM}

Iniciativas legislativas que tornem compulsório o controle metrológico de EEM durante sua vida útil são extremamente urgentes, mas um desafio se evidencia. Ainda não existe uma rede de laboratórios capacitados para a calibração e ensaio de EEM que seja suficiente para atender às demandas no país.

A ANVISA criou a Gerência Geral de Laboratórios de Saúde Pública (GGLAS) objetivando a criação de mecanismos que garantam análises de qualidade para obtenção de resultados laboratoriais confiáveis na área da saúde. A GGLAS, que organiza e estimula o desenvolvimento da capacitação laboratorial no País, definiu uma metodologia de habilitação de laboratórios na Rede Brasileira de Laboratórios Analíticos em Saúde (REBLAS). Até o momento a habilitação dos laboratórios da rede é realizada em três categorias: REBLAS Analítico, REBLAS Equivalência Farmacêutica e REBLAS Ensaio de Proficiência; sendo baseada em dois princípios básicos: capacidade laboratorial e sistema da qualidade. Recentemente a GGLAS realizou uma pesquisa para traçar um diagnóstico da capacidade técnica laboratorial do país referente a EEM. Atualmente, laboratórios para calibração e ensaio de EEM já podem ser habilitados pela ANVISA na modalidade REBLAS Analítico, tendo como requisitos a ABNT NBR ISO/IEC 17025 e/ou Boas Práticas de Laboratório 
(BPL). Até o momento, apenas um laboratório para calibração e ensaio de EEM, localizado no Rio Grande do Sul, se encontra habilitado na REBLAS. O referido laboratório foi habilitado para a realização de ensaios eletromagnéticos em EEM [17].

A seguir é apresentada uma abordagem sobre a importância de profissionais de engenharia clínica nas atividades dos EAS que objetivam a garantia da confiabilidade metrológica de EEM.

\section{A ENGENHARIA CLÍNICA E A CONFIABILIDADE METROLÓGICA DE EEM}

O gerenciamento da atualmente tão sofisticada tecnologia médico-hospitalar, com iniciativas adequadas para a garantia da confiabilidade metrológica de EEM, tem como profissional-chave o engenheiro clínico. Tal profissional é responsável pelo processo de incorporação tecnológica, recebimento, instalação, treinamento de operação, manutenção preventiva e corretiva, avaliação de obsolescência e, na última etapa do ciclo de vida do equipamento, pelo descarte deste. A presença da engenharia clínica nos hospitais só pôde ocorrer após a criação desta sub-área da Engenharia Biomédica, em 1992. Ainda hoje, são poucos os hospitais que contam com a atuação de engenheiros clínicos, apesar da grande densidade de complexos EEM nos mesmos, resultando em um evidente risco para a segurança de pacientes e usuários.

A garantia da confiabilidade metrológica de EEM depende, não só da atuação dos engenheiros clínicos com sua formação complementada por conhecimentos em metrologia, mas também da qualidade dos laboratórios de calibração e ensaio comprovada por um processo de acreditação. Existe um reduzido número de laboratórios capacitados no Brasil, sendo necessário o desenvolvimento de uma rede de laboratórios para avaliação metrológica de EEM. Conforme já citado a ANVISA através da REBLAS acaba de implementar esta inciciativa, onde, até então, apenas um laboratório se encontra habilitado [17].

ENGEVISTA, v. 7, n. 2, p. 51-60, dezembro 2005

\section{CONCLUSÕES}

O presente trabalho aponta para evidências sobre a importância da metrologia na saúde, focalizando a questão dos equipamentos eletromédicos. Ênfase especial foi dispensada à importância da garantia da confiabilidade metrológica dos EEM não somente no momento em que são lançados no mercado consumidor, através da certificação de conformidade exigida pela ANVISA, como também no período de vida útil dos mesmos. Uma ferramenta para o controle pós-comercialização de produtos médicos é a adoção das recomendações da OIML, que ao serem adaptadas para regulamentos técnicos metrológicos resultam na obrigatoriedade de amplo controle metrológico, incluindo o período pós-comercialização. O INMETRO já elaborou duas RTM baseadas em recomendações da OIML. No entanto, mesmo que INMETRO adotasse todas as recomendações publicadas até então pela OIML, esta totalidade não abrange todas as demandas referentes a EEM. De qualquer forma, a regulamentação metrológica na área da saúde é excessivamente importante e necessita não só do surgimento de novas recomendações, como também da realização de estudos para cada vez melhor definir as incertezas recomendáveis, considerando as implicações terapêuticas e diagnósticas na área biomédica. O recente reconhecimento internacional da importância do controle metrológico de EEM na fase pós-comercialização pode ser evidenciado pelo anúncio de normas IEC a serem lançadas contendo procedimentos para a realização de ensaios de EEM em uso.

Como alternativa que vem funcionando para a garantia da qualidade de EEM em uso existem as acreditações dos diversos EAS, que, apesar de voluntárias, têm se revelado excelentes promotoras da confiabilidade metrológica destes equipamentos. Tais acreditações, no entanto, são bastante dependentes da cultura metrológica, aspecto que deve ser disseminado com urgência na área da saúde. 
Outro fator importante para a garantia da confiabilidade metrológica de EEM se refere à elaboração de normas com prescrições para avaliação de desempenho. Em geral as normas existentes abordam somente aspectos de segurança. É muito positivo o fato de que a única norma publicada da série IEC 60601-3, com prescrições para desempenho de EEM, já tenha sido incorporada pela ABNT (NBR IEC 60601-3-1).

Conforme o alerta apontado pela $55^{\mathrm{a}}$ Assembléia Geral da OMS, investimentos na qualificação técnica de recursos humanos envolvidos com EEM podem contribuir para a melhoria da situação dos países em desenvolvimento ou subdesenvolvidos [11]. Fica evidente a necessidade não só da adequada formação dos profissionais de saúde que gerenciam e operam os EEM, com treinamento e cultura metrológica apropriada; como também o apoio essencial para os EAS de uma infraestrutura de engenharia clínica com conhecimentos na área de metrologia; além do apoio de laboratórios acreditados para a realização de calibrações rastreadas e ensaios de EEM. Quanto aos laboratórios, este é um item que constitui um importante desafio, já que não existe uma rede laboratorial capacitada suficiente para atender às demandas no país.

A garantia da confiabilidade metrológica dos EEM, assim como produtos médicos de uma forma geral, seja por força da regulamentação específica ou devido à acreditação do estabelecimento sanitário, pode resultar em prevenção à ocorrência de EA e reduzir de forma significativa os índices de custo e morbi-mortalidade na área da saúde.

\section{REFERÊNCIAS}

[1] "Application of a Single-channel SQUID Magnetometer for Non-Invasive Study of Cardiac Tachyarrhythmias Mechanisms"

E. Costa Monteiro, C. H. Barbosa, E. A. Lima, P. H. Ornelas, E. G. Cavancalti, S. F. Santos, P. Costa Ribeiro. Physica C 354: (1-4) 83-86 (2001)

ENGEVISTA, v. 7, n. 2, p. 51-60, dezembro 2005
[2] "Development of a Low Intensity Magnetic Field Transducer for Biomedical Application based on the Giant Magnetoimpedance Effect”

F. Pompéia, L. A. P. Gusmão, C. H. Barbosa, E. Costa Monteiro, L. A. P. Gonsalves, F. L. A. Machado. Aceito para publicação no Proceedings of the Joint International IMEKO 2005

[3] Brennan TA, Leape LL, Laird NM, et al. Incidence of adverse events and negligence in hospitalized patients: results of the Harvard Medical Practice Study I. $N$ Engl J Med 1991;324:370-376.

[4] Complexo Industrial da Saúde: Insumos e Equipamentos de Uso Médico. R. M. V. Gutierrez e P. V. M. Alexandre. BNDES Setorial, Rio de Janeiro, n. 19, p. 119-155, 2004

[5] NIE-DIMEL-006 Procedimento de Verificação de Esfigmomanômetros mecânicos do Tipo Aneróide. 1997

[6] Campbell N.R.C Mackay D. W.

Accurate blood pressure measurement: why does it matter? Can Med Assoc J 161:277278

[7] M.J. Turner, P.C. Kam, A.B. Baker. Metrology in Medicine. Disponível em:

$<$ http://www.personal.usyd.edu.au/ mjturn er/metrology_in_med_msa_2004.pdf $>$. Acesso em 20 jun. 2005.

[8] Waugh JJ, Gupta M, Rushbrook J, Halligan A, Shennan AH. Hidden errors of aneroid sphygmomanometers. Blood Press Monit 2002, 7:309-12 2002.

[9] Instituto Nacional de Metrologia, Normalização e Qualidade Industrial. Esfigmomanômetros. Disponível em $<$ http://www.inmetro.gov.br/consumidor/pr odutos/esfigmo.asp $>$. Acesso em

15 jul. 2005

[10] Rouse A and Marshall T. The extent and implications of sphygmomanometer calibration error in primary care. J Hum Hyperten 2001, 15:587-591

[11] World Health Organization: FiftyFifth World Health Assembly - Quality of care: patient safety. 2002 
[12] L. E. Costa. Panorama Mundial dos Eventos Adversos no Setor de Saúde. Disponível em $<$ http://www.anvisa.gov.br/tecnovigilancia/ material_PANORAMA_MUNDIAL_EVE NTOSADVERSOS.ppt $>$. Acesso em 20 jun 2005

[13] Comissão Nacional de Energia Nuclear. Normas. Disponível em: $<$ http://www.cnen.gov.br/seguranca/normas .asp>. Acesso em 22 jun. 2005.

[14] Normas para projetos físicos de estabelecimentos assistenciais de saúde. Série: Saúde \& Tecnologia. Ministério da Saúde, 1994

[15] C. I. C. Menezes, M. T. Salles, M. A. S. Silva. Uma Ferramenta para melhoria da Qualidade dos Instrumentos da Área da Saúde. METROLOGIA-2003 - Metrologia para a Vida Sociedade Brasileira de Metrologia (SBM). p. 1-7. 2003

[16] Instituto Nacional de Metrologia, Normalização e Qualidade Industrial. Grupos de Trabalho de Regulamentação Metrológica. Disponível em:

$<$ http://www.inmetro.gov.br/metlegal/comi ssoestec.asp> Acesso em 15 jun. 2005.

[17] Agência Nacional de Vigilância Sanitária.Rede Brasileira de Laboratórios Analíticos em Saúde. Laboratórios e Centros Habilitados. Disponível em:

$<$ http://www.anvisa.gov.br/reblas/bio/anali/ index.htm\#RS>. Acesso em 09 Jun. 2005 